\title{
Integrated modeling and dynamics simulation for the Next Generation Space Telescope (NGST)
}

\author{
Olivier L. de Weck $^{* a}$, David W. Miller ${ }^{\mathrm{a}}$, Gregory J. Mallory ${ }^{\mathrm{a}}$ and Gary E. Mosier ${ }^{\mathrm{b}}$ \\ ${ }^{a}$ Massachusetts Institute of Technology, 77 Massachusetts Avenue, Cambridge, MA 02139,USA \\ bASA Goddard Space Flight Center, Greenbelt, MD 20771, USA
}

\begin{abstract}
NGST represents a challenging problem from the point of view of maintaining a milli-arcsecond level pointing accuracy and diffraction limited wavefront performance in the presence of dynamic onboard disturbances during science observations in a cryogenic environment. A Dynamics-Optics-Controls-Structures framework is being developed in support of the NGST dynamics and controls modeling program. First, an integrated model comprising multiple disturbance sources, structures, optics and control systems was developed in order to predict the expected dynamic wavefront error and line-of-sight jitter. A disturbance analysis was carried out using frequency domain and Lyapunov techniques. An indexing technique was subsequently used to analyze the sensor-actuator topology of the system. A sensitivity analysis revealed which modal parameters contribute significantly to the root-mean-square errors. The critical frequencies were found to be in the range from $5-50 \mathrm{~Hz}$. This information was subsequently used to recommend performance improvements including stiffening the secondary mirror tower, isolating reaction wheels and adding passive damping treatments. The combination of improvements was implemented numerically, resulting in good performance with sufficient design margins. Isoperformance analysis was introduced as a means of trading system parameters, while holding the performance constant. This research is motivated by the fact that it will not be possible to test the fully deployed observatory in a $1 \mathrm{~g}$ environment before launch.
\end{abstract}

Keywords: NGST, dynamics, optics, controls, structures, simulation, integrated modeling, disturbances

\section{INTRODUCTION}

\subsection{Problem Formulation}

During science observations ${ }^{1}$ NGST requires line-of-sight (LOS) stabilization to 4.8 milli-arcseconds (mas), $1 \sigma$, and a rootmean-square (RMS) wavefront error (WFE) of less than $\lambda / 14^{2}$, which corresponds to $0.157 \mu \mathrm{m}$ at a wavelength of $2.2 \mu \mathrm{m}$. The path from disturbances $w$ to the performance metrics of interest $\mathrm{z}$ must therefore be modeled in detail (Figure 1). At the diffraction-limited wavelength of $2.2 \mu \mathrm{m}$ the full width at half maximum of an image is 51 mas. Guiding errors must be less

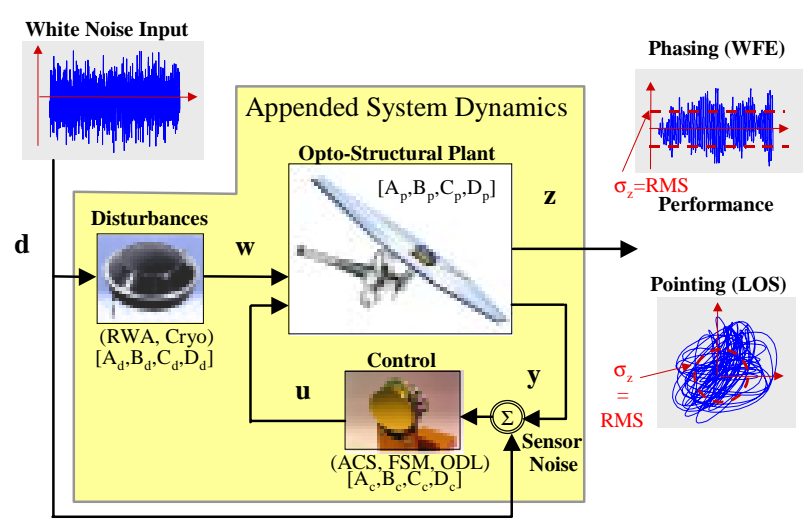

Figure 1: NGST Yardstick Dynamics \& Controls Diagram. RWA picture: http://www.ithaco.com, FSM picture: http://www.lefthand.com/prod_fsm.html than $10 \%$ of this value (i.e. 5 mas) to be practically negligible. It is assumed that the system is linear and time invariant (LTI). Three disturbances sources (reaction wheel assembly, cryocooler, guide star noise) are acting simultaneously during the fine pointing observation mode as zero-mean random stochastic processes. This ignores the ACS sensor and FSM inner loop noise. The effect of the three noise sources is captured with the help of state space pre-whitening filters $\left[A_{d}, B_{d}, C_{d}, D_{d}\right]$, such that the input to the appended system dynamics $\left[A_{z d}, B_{z d}, C_{z d}, D_{z d}\right]$ is assumed to be unitintensity white noise $d$, which is uncorrelated for each disturbance source. The shaped disturbances $w$ are then propagated through the opto-structural plant dynamics $\left[A_{p}, B_{p}\right.$ $\left.{ }^{,} C_{p}, D_{p}\right]$. A compensator $\left[\begin{array}{llll}A_{c}, & B_{c}, & C_{c}, & D_{c}\end{array}\right]$ stabilizes the observable rigid body modes with an attitude control system (ACS) and improves the pointing disturbance rejection

\footnotetext{
${ }^{*}$ Correspondence: email: millerd@ mit.edu; WWW: http://web.mit.edu/ssl ; Telephone: (617) 253-3288

${ }^{1}$ The slew mode for the acquisition of new science targets was analyzed in a preliminary study [1].

${ }^{2}$ In reality only a fraction of the total $\lambda / 14$ error budget will be allocated to jitter (probably $<\lambda / 20$ ).
} 
capability with a fast steering mirror (FSM) loop. The outputs $y$ of the fine guidance sensor (FGS) for the angular guide star position are subject to noise. The goal of the study is to predict the RMS values and sensitivities of the performances $z_{1}$ (wavefront error) and $z_{2}$ (LOS jitter) given the above assumptions. If margins are insufficient, promising improvements to the system dynamics shall be suggested and implemented numerically.

The NGST "Yardstick" design used in this paper was proposed by a GSFC-led study team in 1996 [3]. The main observatory components are the Spacecraft Support Module (SSM), the large inflatable sunshield (ISS), the optical telescope assembly (OTA) and the integrated science instrument module (ISIM). The ISIM houses all the detectors, cameras, cryocoolers, the deformable mirror (DM) ${ }^{3}$, a fast steering mirror (FSM) for LOS stabilization and all other optical elements. NGST will use the ACS for coarse pointing only. The remaining pointing error will be sensed using the near-infrared (NIR) camera itself.

\subsection{The DOCS framework for analysis and design}

A number of tools have been developed as part of the DOCS (Dynamics-Optics-Controls-Structures) framework for precision opto-mechanical space systems. Within the MATLAB ${ }^{\mathrm{TM}}$ environment a model of the spacecraft can be created, which simulates the dynamic behavior of the structure, the optical train, the control systems and the expected disturbance sources in an integrated fashion. Figure 2 shows a block diagram of DOCS. The existing toolboxes are compatible with IMOS (version $4.0)^{4}$, MSC/NASTRANTM as well as DynaMod and DynaCon ${ }^{5}$. Once an initial model has been created and numerically conditioned, the root-mean-square (RMS) values of opto-mechanical performance metrics of the system (e.g. pathlength difference, pointing jitter, fringe visibility, null depth) can be predicted. The exact sensitivities of the RMS with respect to modal or physical design parameters can be computed. The goal of the uncertainty analysis is to associate error bars with the predicted RMS values, which are based on an uncertainty database resulting from past ground and flight experience. The actuator-sensor topology of the system can be analyzed numerically to ensure that the control system uses the actuator-sensor pairs that will ensure maximum disturbance rejection or tracking performance. Once a design has been found that meets all

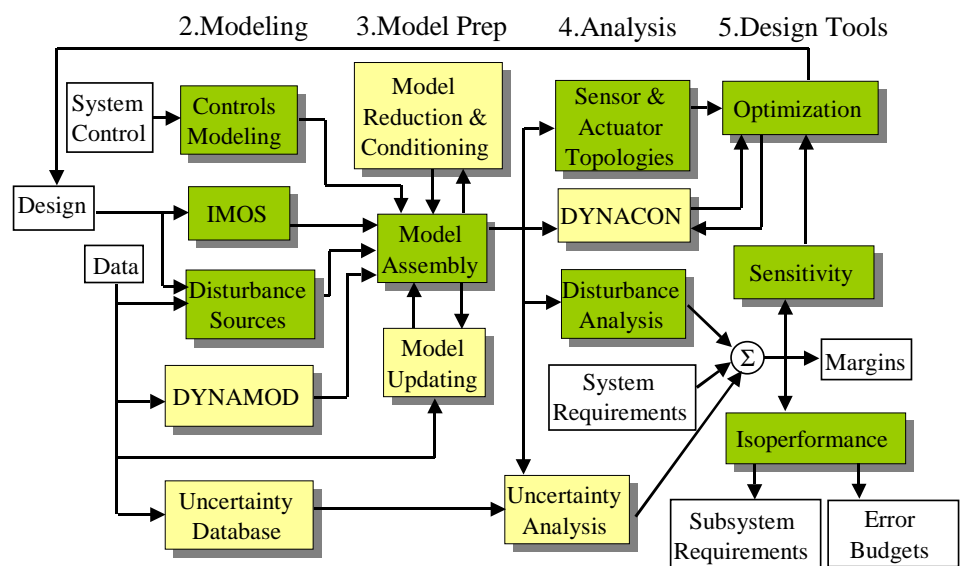

Figure 2: DOCS block diagram. Note: The sections in this paper correspond to the column headings in this diagram requirements with sufficient margins, an isoperformance analysis can be conducted. Treating the performance as a constraint, the expected error sources (error budgeting) or key design parameters (subsystems requirements definition) can be traded with respect to each other. If hardware exists, the experimental transfer functions can be used to update the structural, avionics and uncertainty models throughout the life of the program to achieve a convergent design that will achieve mission success. Preliminary versions of the framework have been successfully applied to conceptual designs of the Space Interferometry Mission (SIM), the Terrestrial Planet Finder (TPF) and Nexus. The following sections present the results obtained for NGST corrsponding to dark shaded boxes in Figure 2.

\subsection{Previous work}

Early studies of potential architectures and conceptual designs of the Next Generation Space Telescope have been carried out since 1996 [5]. A paper on wavefront sensing and control for NGST was published by Redding, Bely, Burg and coworkers [6]. Further papers are by Mosier, Femiano and Ha on integrated modeling for NGST [7], derivation of an optimal control law for spacecraft slews [8], and fine pointing control for NGST [29]. Kissil prepared a number of internal memos on the various finite element models of the yardstick design [9], which were used as a basis for structural modeling in this paper. The fundamental work that allows formulating the linearized models of complex optical systems in state space form was performed by Redding and Breckenridge [10]. Bialke published papers on the sources of reaction wheel disturbances [11]. Masterson developed a methodology for analyzing reaction wheel disturbance test data and deriving empirical and physical models [21]. Cryocooler disturbances were analyzed by Castles e.a. [12], as well as Collins [13]. Finally this work builds on the developments by Gutierrez [14], who describes the fundamentals of the disturbance and sensitivity analysis framework and a more comprehensive NGST analysis by de Weck [2], whose results are summarized in this paper.

\footnotetext{
${ }^{3}$ The DM is used for quasi-static wavefront control, but not as a high-frequency actuator.

${ }^{4}$ Available for academic licensing from the Jet Propulsion Laboratory (JPL) [15].

${ }^{5}$ Available on a commercial basis from Midé Technology Corporation, Cambridge, MA 02142, U.S.A.
} 


\section{INTEGRATED MODELING ${ }^{6}$}

The NGST systems level block diagram in Figure 3 approximates the relevant dynamics with LTI state space systems in continuous time. The system boundaries have been chosen such that they do not include the external environment (e.g. solar pressure, heat flux). These interactions are simplified by assuming that the inputs to the system are the RWA disturbances, cryocooler vibrations and the fine guidance sensor (FGS) noise directly. The two dynamic outputs of interest are the WFE $\left(z_{1}\right)$ and the LOS jitter $\left(z_{2}\right)$. The following subsections describe the models which constitute each major block in Figure 3.

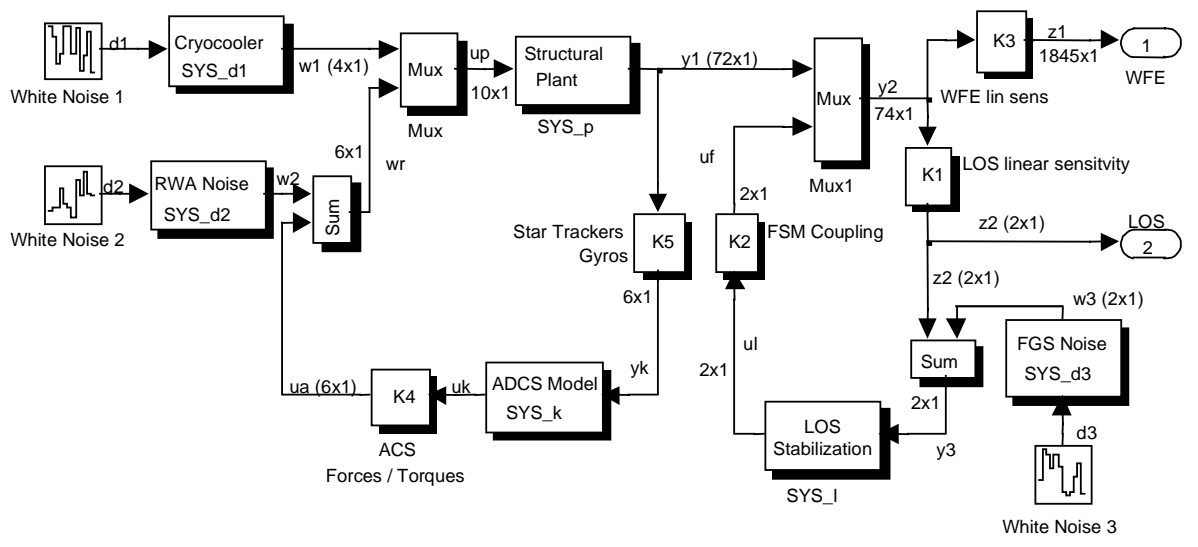

Figure 3: NGST continuous time LTI integrated model block diagram

\subsection{Structural Plant Dynamics (SYS_p)}

The structural dynamics modeling task for NGST consists in modeling the Yardstick design with its main structural members such as plates, beams, rods, concentrated masses, generalized stiffnesses and rigid body elements. A simplified finite element model was developed which contains 109 grid points and 573 active degrees-of-freedom and is based on previous work by Kissil [9]. The model is intended for trade studies since it only captures the overall dynamics of the system. The inputs $u_{p}$ (10x1) to the FEM are the forces and torques from the cryocooler (4x1) entering at the ISIM and from the reaction wheels $(6 \times 1)$ entering at ACS grid point. The FEM output $y_{1}(72 \times 1)$ comprises 11 optical grid points, which represent 66 degrees of freedom. The additional 6 degrees of freedom are the 3 rotations and 3 angular rates of the ACS grid point. We solve the generalized eigenvalue problem $\left[K-\lambda_{i} M\right] \phi_{i}=0$, where $M$ and $K$ are the global mass and stiffness matrix respectively, which have been previously assembled in IMOS [15]. Figure 4 shows a mosaic of four interesting modes of the finite element model. The first flexible mode is expected to be due to the ultra-light sunshield and is predicted to occur at $0.29 \mathrm{~Hz}$. Other significant modes are the first bending mode of the secondary mirror support blades at $3.4 \mathrm{~Hz}$ and the first global pitch mode involving the primary mirror at $7.38 \mathrm{~Hz}$. The state-space matrices are assembled by assuming modal damping of $\zeta=0.001$ for the entire structure.

\subsection{Linear Optics Sensitivity Matrices $\left(K_{1}, K_{3}\right)$}
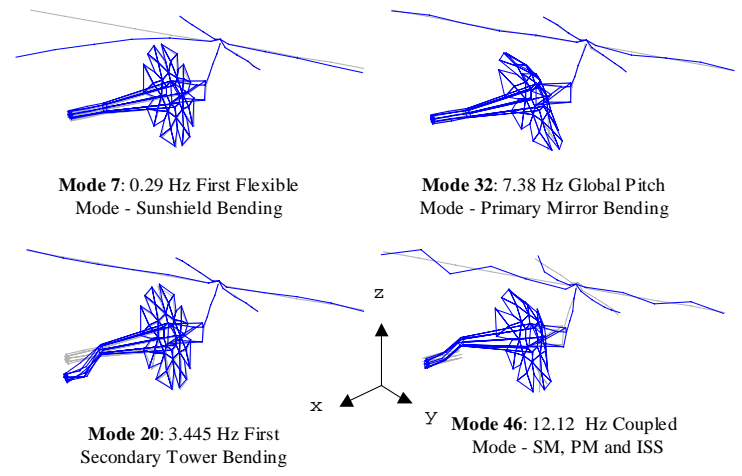

Figure 4: Mosaic of four NGST FEM normal modes. Modes 1-6 correspond to the rigid body modes.

The yardstick utilizes nine deployable segments in a symmetric pattern to form its primary mirror (PM). The secondary mirror (SM) is mounted on a deployed 4-strut tower, attached to a conical baffle. Assuming the individual petals are rigid bodies one representative grid point can be chosen for each PM petal and the center petal. Furthermore we read the displacements of the ISIM, which contains the FSM, DM, cryocooler and the focal plane, and the node located at the apex of the SM. The linear sensitivity matrices for the optics model are computed by introducing a unit perturbation to one degree of

\footnotetext{
${ }^{6}$ See first column in DOCS block diagram of Figure 2.
} 
freedom at a time and computing wavefront and centroid in MACOS [24]. The matrices are thus formed one column at a time by numerical differentiation. Mathematically, the linear optics models are given by:

$$
\begin{aligned}
& \text { Wavefront error: } z_{1}=W_{o}+\frac{\partial W}{\partial y_{2}} y_{2}=K_{3} y_{2} \\
& \text { Centroid error: } z_{2}=C_{o}+\frac{\partial C}{\partial y_{2}} y_{2}=K_{1} y_{2}
\end{aligned}
$$

where $y_{2}(74 \times 1)$ is the vector of translation and rotations of the FEM coordinates $(72 \times 1)$, augmented by the FSM gimbal coordinates $(2 \mathrm{xl})$, $C$ is the centroid for the chief ray $(2 \mathrm{xl}), W$ is the WFE vector $(1845 \mathrm{xl}), \quad K_{1}=\partial C / \partial y_{2}$ is the centroid linear sensitivity matrix (2x74), and $K_{3}=\partial W / \partial y_{2}$ is the wavefront linear sensitivity matrix (1845x74). We assume that $W_{o}=0$ and $C_{o}=0$, which means that we are not considering the static components of wavefront and centroid error $^{7}$. A plot of the linear sensitivity matrix $K_{l}$ for centroid versus the degree of freedom number of the plant output vector is shown in Figure 5. It shows that the centroid is particularly sensitive to the $y$ and $\mathrm{z}$ rotations of the central petal mirror node in the spacecraft coordinate axes shown in Figure 4. Even though the ray-tracing

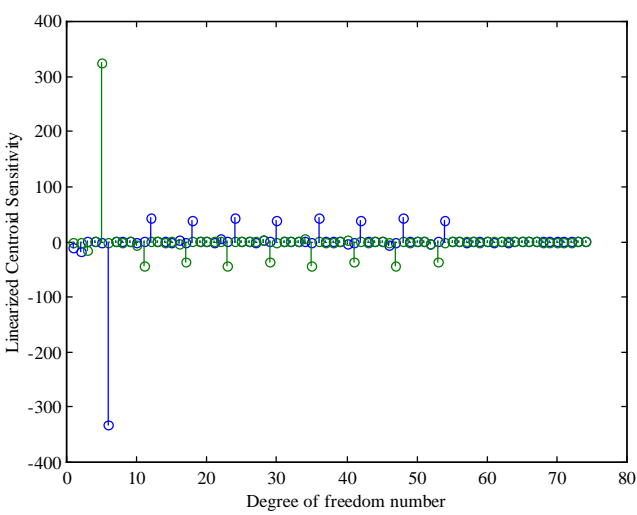

Figure 5: Graph of centroid linear sensitivities in $\mathrm{x}$ and $y$ detector axes as a function of FEM degree-offreedom. Note: These sensitivities evolve continously with changes in the optical prescription of NGST. algorithm in MACOS allows a good representation of the spatial distribution of the WFE, the disturbance analysis condenses the results down to only two scalar numbers: RMS WFE and RMS LOS. These are obtained from the 1847 outputs as :

$$
z_{\text {WFE RMS }}=\left(\frac{1}{n_{\text {rays }}}\left(z_{1}^{T} z_{1}\right)\right)^{1 / 2} \quad z_{\text {WFE LOS }}=\left(\frac{1}{2}\left(z_{2}^{T} z_{2}\right)\right)^{1 / 2}
$$

Thus the WFE RMS value is the root mean square result of the values for each individual ray across the physical aperture of the telescope. In a similar manner the RMS LOS is calculated from the centroid $\mathrm{x}$ and $\mathrm{y}$ errors (in detector axes).

\subsection{Cryocooler Disturbance Model (SYS_d1)}

The cryocooler on NGST will provide the necessary active cooling for the MIR SiAs-detectors down to about $\sim 7-8 \mathrm{~K}$ during observations in the wavelength range from 2-16 $\mu \mathrm{m}$ [5]. A Stirling cryocooler is modeled in this study due to the availability of vibration test data. The vibration levels of many cryocoolers differ by orders of magnitude, depending on the type and exact configuration as demonstrated by Castles and co-workers [12],[19]. Figure 6(a) shows the Bae80K cooler that was chosen for this study. The axial force experimental data is shown in Figure 6(b).

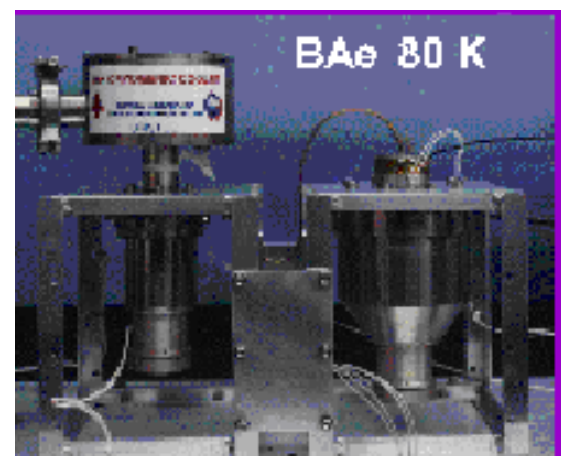

(a)

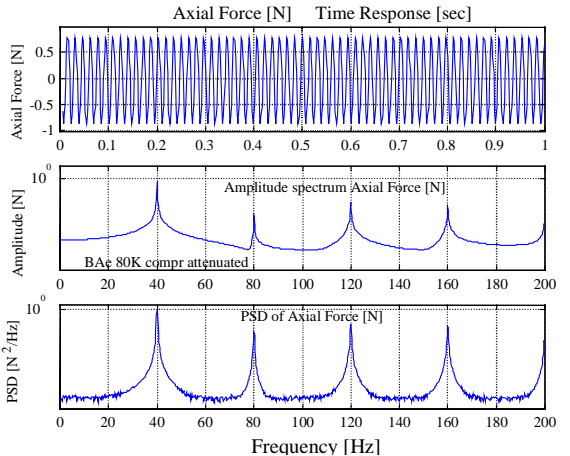

(b)
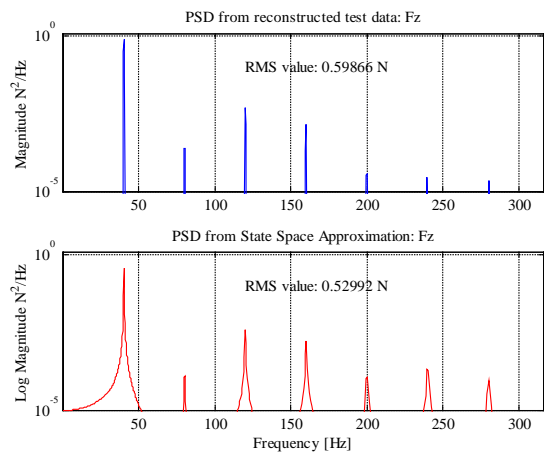

(c)

Figure 6 : (a) Bae $80 \mathrm{~K}$ cryocooler [19]; (b) Reconstructed axial force disturbance, assumes -20 dB (factor 0.1) of attenuation due to vibration suppression measures according to Collins [13] (top), axial force amplitude spectrum (middle), axial force power spectral density function-PSD, (bottom); (c) Comparison of experimental PSD's (top) and $2^{\text {nd }}$ order system approximation (bottom)

The cooling in the Sterling cycle $(40 \mathrm{~Hz})$ is achieved through controlled motion of a piston and a displacer to compress and then expand a working fluid [12]. Vibrations are generated by a momentum imbalance between the linearly reciprocating

\footnotetext{
${ }^{7}$ These static components have been previously shown to be present in the system due to the nominal design, thermal distortions, etc.
} 
elements. The nonlinear gas spring creates the higher harmonics. Experimental vibration data from [21] is converted to PSD and state space representation by approximating the PSD-harmonics with $2^{\text {nd }}$ order lightly damped poles. A state space representation (64 states) of the cryocooler disturbance in Gauss-Jordan form for the $F_{x}, F_{y}, F_{z}$ and $M_{z}$ components is obtained, whereby experimental data shows that $M_{x}$ and $M_{y}$ are negligible [19].

\section{4 Reaction Wheel Disturbance Model (SYS_d2)}

Precision reaction wheels induce disturbance forces and moments, which are created due to static and dynamic imbalances. The imbalances are produced by a non-uniform mass distribution within the flywheel. The static imbalance represents the fact that the center of mass of the flywheel is not exactly on the spin axis. This can be interpreted as a small mass at radius $r$ as shown in Figure 7(a). While the wheel is spinning, this offset mass produces a radial centripetal force, whose magnitude is given as $F_{r}=m r \omega^{2}=U_{s} \omega^{2}$, where $m[\mathrm{~kg}]$ is the offset mass, $r[\mathrm{~m}]$ is the radius of the flywheel and $\omega(\mathrm{rad} / \mathrm{sec})$ is the angular velocity of the wheel. The static imbalance $U_{s}$ is a flywheel mass property and is often given in $[\mathrm{gcm}]$. The dynamic imbalance is representative of the cross product of inertia of the flywheel, which is caused by a slight angular misalignment of the principal moment of inertia with the spin axis. Experimental measurements show that not only the fundamental frequency but that higher harmonics contribute significantly to the disturbance spectrum [20].

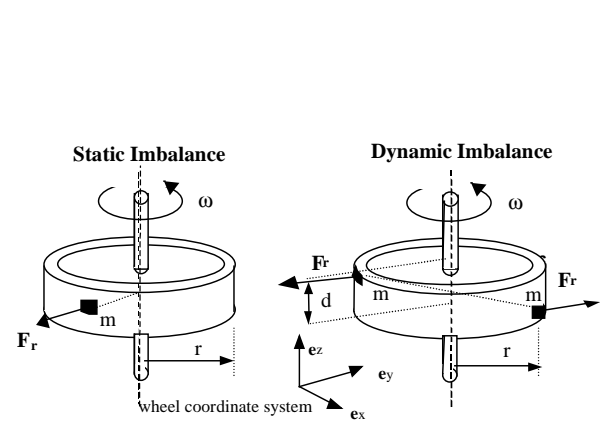

(a)

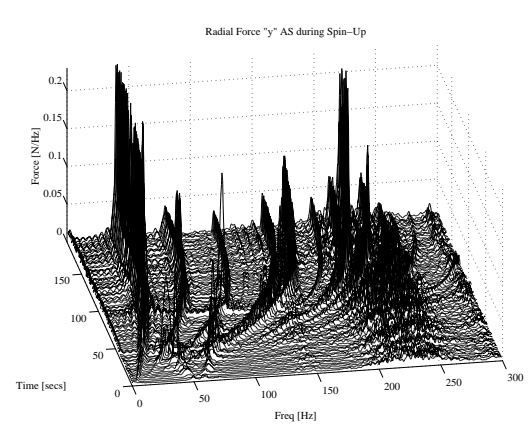

(b)

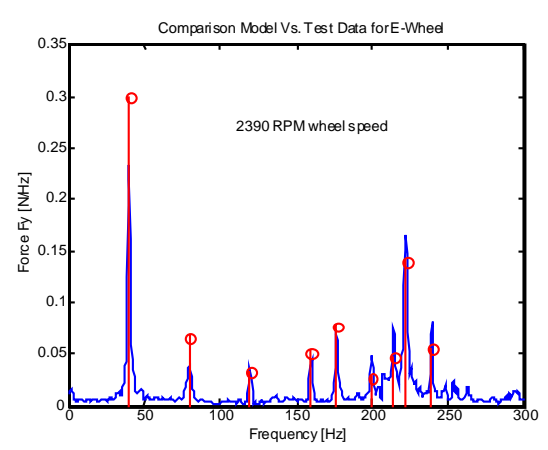

(c)

Figure 7: (a) Schematic representation of static and dynamic imbalance; (b) Waterfall plot for $F_{y}$-component for ITHACO E-Wheel spinup test. Imbalances from E-Wheel test data: $U_{s}=0.7160 \mathrm{gcm}$ (spec: $U_{s}<1.8 \mathrm{gcm}$ ), $U_{d}=29.54 \mathrm{gcm}^{2}$ (spec: $U_{d}<60$ gcm ${ }^{2}$ ), i.e. wheel meets its specification; (c) Comparison of experimental amplitude spectrum and empirical $h_{k}$ 's and $C_{k}$ 's at 2390 RPM wheel speed

The ridges, which emanate from the origin at 0 seconds and $0 \mathrm{~Hz}$ in the waterfall plot of Figure $7(\mathrm{~b})$, represent the harmonics of the wheel speed disturbance. The first ridge, which eventually saturates at $40 \mathrm{~Hz}(=2400 \mathrm{RPM})$ is the fundamental frequency of the wheel in revolutions per second [RPS]. After identification of the harmonics $h_{j k}$ in the test data (Figure $7(\mathrm{c}))^{8}$, the coefficients $C_{j k}$ of these harmonics are numerically determined with a least-squares fitting approach as presented by Masterson [21]. For the E-wheel used in this analysis the dominant harmonics were found to be $h_{k}=1.0,2.0,2.9,3.0,3.88$, 4.0, 4.43, 5.20, 5.40 and 6.0. As indicated by Bialke [11] the values for $C_{j k}$ can change from wheel to wheel and fine-tuning with installed bearings can reduce these values by a factor of 2-5. A trade involving $U_{s}$ will be shown in subsection 5.2.

A reaction wheel assembly (RWA) with four wheels has been chosen for the NGST yardstick design in a square-based pyramidal configuration (Figure 8(a)). The forces and torques in each wheel frame must be transformed into body frame using known coordinate transformations as derived by Gutierrez [22]. Reaction wheel disturbances in this paper are modeled as stochastic processes. This is due to the fact that individual wheel speeds cannot be precisely predicted during an observation. The key random variables are the individual wheel speeds $f_{i}$ and the individual wheel phases $\phi_{i j k}$. This analysis assumes a random wheel phase $\phi_{i j k}$ with a uniform probability density function on the interval $[0,2 \pi]$. The probability density function of the wheel speeds is based on work done by Gutierrez [14] and Melody [20]. The stochastic reaction wheel model can be written as a 6x6 cross spectral density matrix, if we make reasonable assumption about the bias wheel speed $R_{o}$ of each wheel and the expected variation of the wheel speed $d R$ during observations. The 6x6-cross spectral density matrix in the spacecraft frame is the summation of the contributions from the $N$ wheels. The result is the $6 \times 6$ spectral density matrix whose diagonal terms are shown in Figure 8(b). The "saw-tooth" are due to the individual wheel sweeping across the frequency space. While in the case of the cryocooler disturbance we have a narrowband tonal disturbance, the RWA disturbances are rather broadband in nature, when averaged over long time periods. We then introduce linear filters which,

\footnotetext{
${ }^{8}$ This ITHACO E-wheel was an off-the-shelf standard catalog product that had not yet been balanced for minimum vibration operation.
} 
when driven by white noise, 'overbound' the PSDs, while capturing the frequency content and producing a comparable RMS value (see Figure 8(c)).

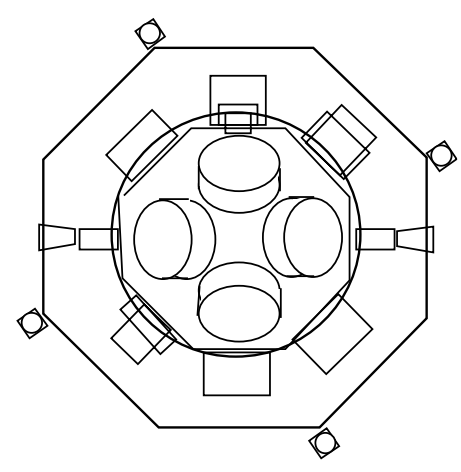

(a)
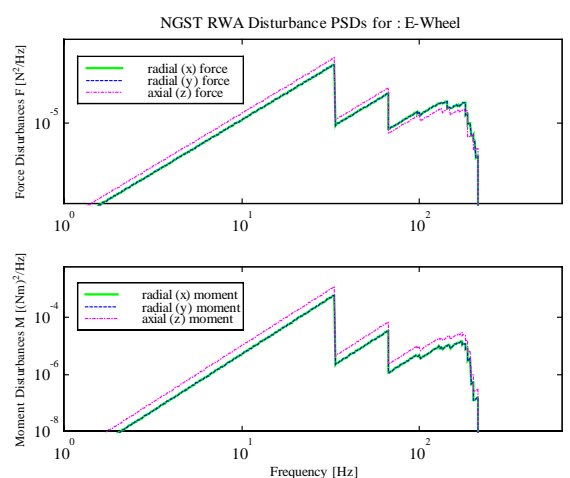

(b)
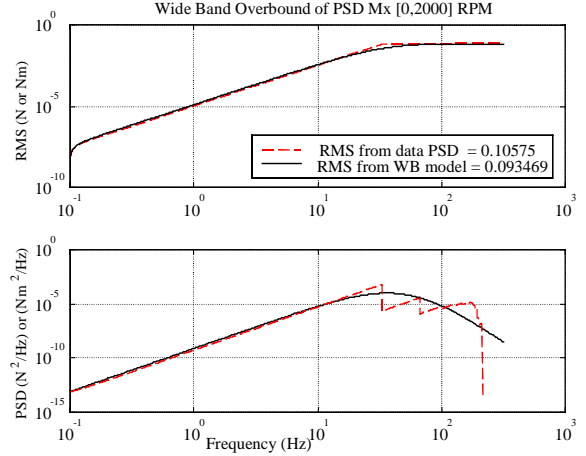

(c)

Figure 8: (a) NGST RWA pyramidal arrangement; (b) RWA disturbance PSD's assuming: ITHACO E-wheel, Number of wheels $\mathrm{N}=4$, Euler angles of reference wheel : $\alpha=-135^{\circ}, \theta=-90^{\circ}, \gamma=0^{\circ}$, Wheel offset distance $: \mathrm{d}=0.5 \mathrm{~m}$, Bias wheel speed : Ro= $1000 \mathrm{RPM}$, wheel speed variation: $\Delta \mathrm{R}= \pm 1000 \mathrm{RPM}$, uniform wheel speed pdf.; (c) PSD overbound approximation of RWA disturbances for the $M_{x}$ component. The RMS of the disturbance PSD is equal to $0.106 \mathrm{Nm}$, whereas the RMS for the wideband model is $0.093 \mathrm{Nm}$.

\subsection{Fine Guidance Sensor Noise(SYS_d3)}

It is planned to use the NIR science camera itself as the fine guidance sensor (FGS) for NGST. Once a guide star in the science field is identified, a small (10x10) window of pixels surrounding the star is addressed at a high frame rate (10-100 frames per second). This data is passed to a centroider, which generates commands for the FSM. The guiding sensor must be able to guide on stars of about 16.5 magnitude in the I band. These relationships were first explored by Bely and coworkers for application to NGST [4]. The measuring error of a stellar guiding sensor is essentially due to photon noise (and pixel quantization) and is characterized by its noise equivalent angle (NEA), which can be expressed as:

$$
\mathrm{NEA}=\frac{1}{\mathrm{k} \sqrt{\mathrm{N}}} \cdot \sqrt{\left(1+\frac{\mathrm{R}_{\mathrm{o}}}{\mathrm{N}}\right)+\varepsilon_{\mathrm{cent}}^{2}}
$$

where $k$ is the slope of the centroid transfer function, $N$ is the total number of photoelectrons, $R_{o}$ is the detector readout noise, and $\varepsilon_{\text {cent }}$ is the centroiding error. The total number of detected photoelectrons $N$ in turn is defined by the following equation: $N=T_{r} \cdot Q E \cdot A \cdot B P \cdot 10^{-0.4 \cdot M} \cdot P_{H} \cdot T_{I N T}$, where $T_{r}$ is the optical transmissivity, $Q E$ is the detector quantum efficiency, $A$ is the sensor aperture, $B P$ is the freq. bandpass, $M$ is the guide star magnitude, $P_{H}$ is the number of collected photons per $\mathrm{m}^{2}$ per $\mu \mathrm{m}$ per sec. and $T_{I N T}$ is the integration time. The plot in Fig. 9(a) gives the NEA as a function of $T_{I N T}$.

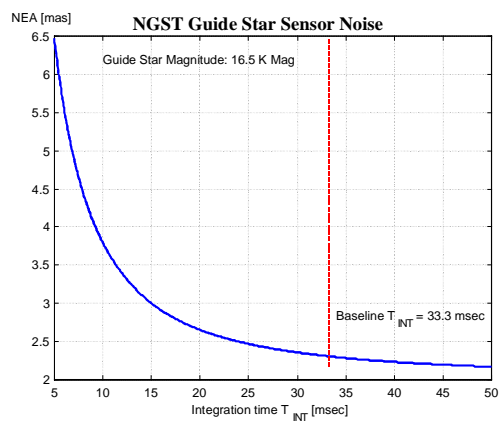

(a)

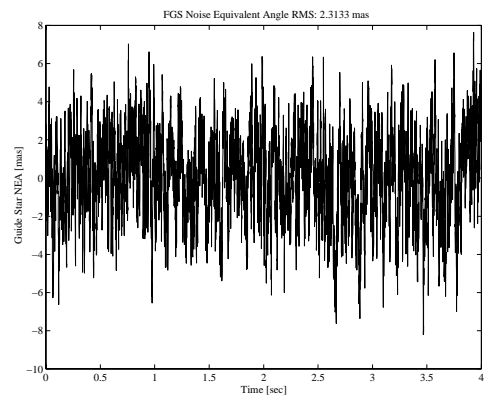

(b)

$$
\begin{aligned}
& \sigma_{N E A}^{2}=\frac{1}{2 \pi} \int_{-\infty}^{+\infty} \underbrace{\frac{k_{N E A}^{2}}{\omega^{2}+\omega_{F G S}^{2}} d \omega=}_{S_{w w}} \\
& \frac{k_{N E A}^{2}}{\pi \omega_{N E A}}[\underbrace{\arctan (\infty)}_{\pi / 2}-\underbrace{\arctan (0)}_{0}]=\frac{k_{N E A}^{2}}{2 \omega_{N E A}}
\end{aligned}
$$

(c)

Figure 9 : (a) Guide star sensor noise in terms of NEA as a function of the sampling time $T_{I N T}$ in milliseconds for a read noise of 30 electrons, quantum efficiency of 0.8 , an 8 meter diameter telescope, wavelength of 2.2 microns with a $+/-25 \%$ bandpass, a throughput of 0.6, and a guide star of K magnitude 16.5. (b) Sample realization of single channel of FGS noise with RMS=2.31 mas corresponding to the baseline integration time of $33.3 \mathrm{msec}$ in (a); (c) Equation (4) for computing RMS of FGS noise based on LPF. 
The current baseline establishes an integration time of $33.3 \mathrm{msec}$. In order to use the FGS noise model for the disturbance analysis it is necessary to convert the NEA to an equivalent PSD and state space representation. We can approximate the discrete-sampling with a continuous low-pass filter (LPF), whose corner frequency is equal to the update frequency $\omega_{F G S}$. The variance of the FGS noise can be computed from the area under the PSD as shown in Figure 9(c). We thus obtain a value for $k_{N E A}$, since $\omega_{F G S}$ is known a priori. Finally we cast the FGS noise into canonical state space form.

\subsection{Controls Modeling (SYS_k, SYS_l)}

Previous work by Mosier, Femiano and Ha [18] resulted in a high-fidelity model of the ACS for NGST. This controller featured decoupled roll/pitch/yaw PID loops and was designed using classical control techniques. For use in this analysis, however, we will employ a MIMO ACS controller that is based on the LQG (Linear Quadratic Gaussian) regulator. A Riccati equation is solved to obtain the matrix of optimal controller gains in an $\mathrm{H}_{2}$-sense (SYS_k). Frazzoli initially developed this approach for use on the conceptual TPF mission design [25]. For NGST the pointing accuracy of the spacecraft itself and the fine pointing requirements of the optics will be decoupled within the dynamic range of the fast steering mirror (FSM). The large collecting aperture of NGST permits the sensing of line-of-sight variations at high rates using relatively faint guide stars. The OTA itself can be left to wander slightly due to SSM-induced pointing errors [7]. The SSM inertial reference sensors (star trackers (ST) and/or coarse sun sensors(CSS)) are not suitable as a tracking reference for the FSM for two reasons. First, the ACS sensors are non-collocated with respect to the centroid, which can introduce nonminimum phase zeros and create stability problems for the FSM loop. Secondly the resolution of the ST and CSS's is insufficient to meet the NGST fine pointing requirements. Figure 10 shows the model for the FMS control, used in this paper and developed at GSFC. We assume that the FSM plant dynamics are much higher than the plant dynamics of the spacecraft structure, so that the FSM plant

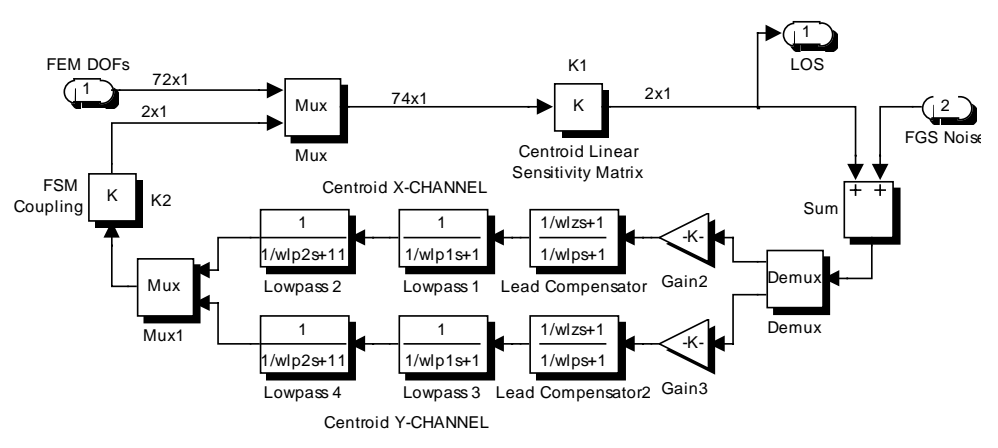

Figure 10: FSM controller in block diagram form (both channels)

itself is not modeled. The FSM model was derived from transfer function data taken from an off-the-shelf design [18]. The FSM acts as a low-pass filter to the mirror angle command, including guide star noise, but as a high-pass filter to the base motion. The transfer function representation from Figure 10 is transformed into a state space representation, which contains both parallel channels in the X and Y axes thus yielding the FSM controller (SYS_l).
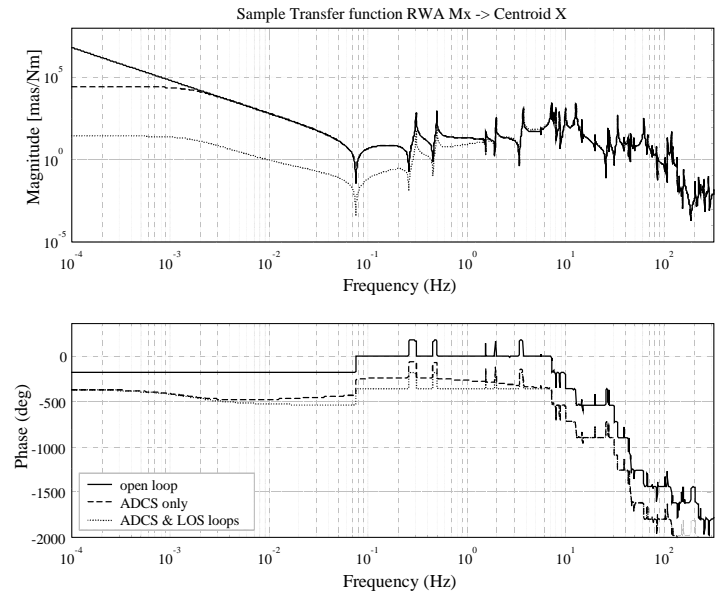

Figure 11: Comparison of open loop, ADCS only and all loops closed transfer functions from RWA $M_{x}$ to centroid $\mathrm{x}$ for NGST.

shows a sample transfer function from the RWA component $M_{x}$ at the at the RWA input node to the centroid $x$ channel. The first transfer function from the top in the magnitude portion of the Bode plot corresponds to the open loop case. The rigid
The disturbance analysis (Lyapunov approach) requires that the system be written in closed loop state space from from white noise input $d$ to the performance metrics of interest $z$. The frequency-domain based disturbance analysis also allows the system to be written from the actual (shaped) disturbances $w$ to the performances $z$. The goal of model preparation is to assemble all submodels into an overall model, to check the stability of that model and to verify that all the units are correct. Also model balancing and reduction are recommended in order to speed up computations and remove numerical illconditioning. The first step is to write the overall state vector in appended form. The state vector is typically written as $q=\left[\begin{array}{lll}q_{d} & q_{p} & q_{c}\end{array}\right]^{T}$, where $q_{d}$ are the disturbance filter states, $q_{p}$ are the (structural) plant states and $q_{c}$ are the controller states. In the NGST baseline analysis case we have a total of 451 states in the appended state vector. The overall state space system relates the three statistically independent white noise sources $d_{1}, d_{2}$ and $d_{3}$ to the performance metrics z. Figure 11
$M_{x}$ at the at the RWA input node to the centroid $x$ channel. The 
body mode behavior at low frequency can clearly be seen. This means that the rigid body mode that is controlled by $M_{x}$ has its poles on the $j \omega$-axis (neutrally stable) and requires ACS stabilization. This is achieved by closing the loop on the ACS controller (dashed line). It can be seen that the controller is low bandwidth $(\sim 0.025 \mathrm{~Hz})$, but that it provides a first level of stabilization for the centroid $\mathrm{x}$. A second level of attenuation is provided by the fast steering mirror (FSM) loop, which is limited in its effective bandwidth. The attenuation of input disturbances is effective up to a bandwidth of roughly $2 \mathrm{~Hz}$. Higher frequency modes are not attenuated, which might indicate that vibration mitigation measures will be needed. The LOS stabilization loop provides about $50 \mathrm{~dB}$ attenuation at $0.01 \mathrm{~Hz}$.

\section{SYSTEM ANALYSIS}

\subsection{Disturbance Analysis}

The goal of this section is to predict the dynamic performance of the observatory in terms of wavefront error (RMS WFE) and LOS jitter (RMS LOS) given the model assumptions made in the previous sections. Additionally we want to identify critical disturbances and modes that drive system performance. The frequency-domain analysis starts with the PSD's of the disturbances and computes the PSD's of the performance metrics. The covariance is obtained by computing the integral under the area of the performance PSD's. Secondly a steady-state Lyapunov equation is solved on the appended closed loop system assuming that the input is white noise. Details on these techniques are contained in references [14] and [23].

The Frequency domain analysis for NGST is carried out with the model that represents the closed loop system from $w$ to $z$ with the ACS and LOS stabilization loops closed. This system is then injected with the 12 components of shaped noise (4 cryocooler, 6 reaction wheel assembly and 2 FGS noise components) in order to obtain the two performance metrics of interest: RMS LOS and RMS WFE. The results for the frequency domain analysis for RMS WFE are shown in Figure 12.

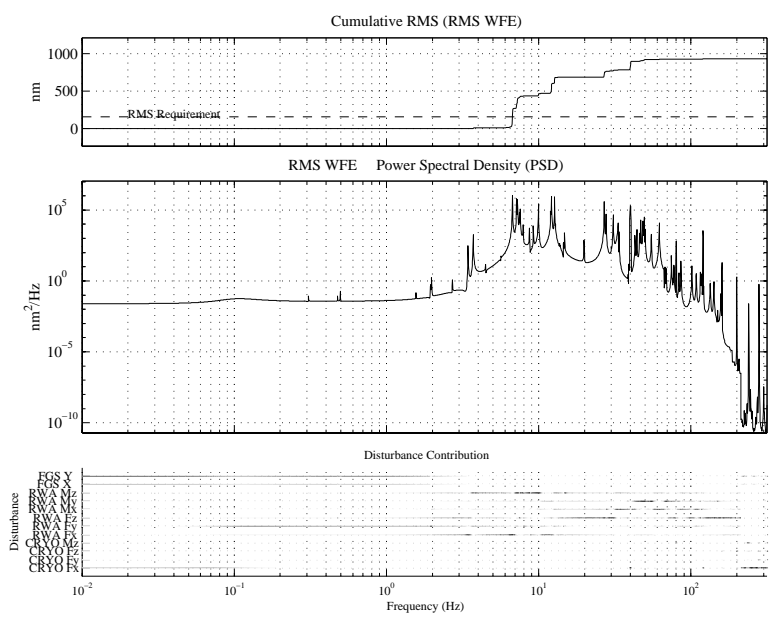

(a)

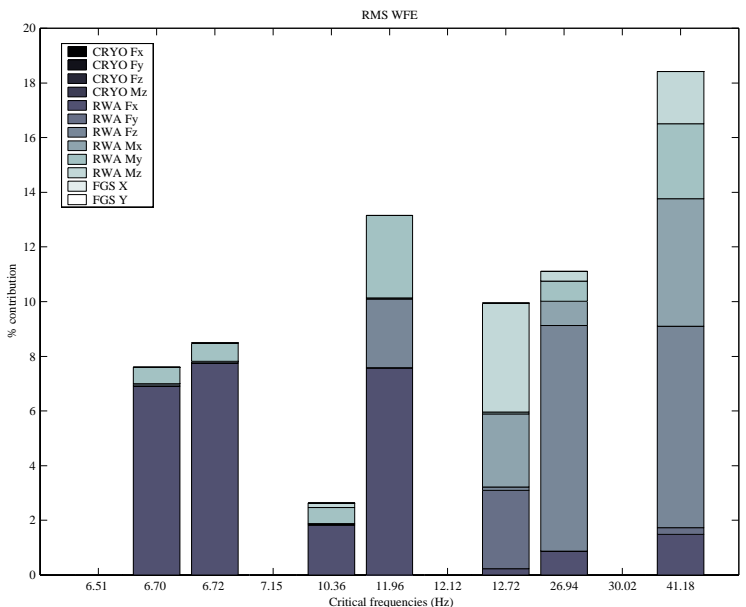

(b)

Figure 12: (a) PSD Analysis nominal results for WFE; (b) Modal significance chart for WFE

The middle plot of Figure 12(a) shows the performance PSD for WFE, the top plot shows the cumulative RMS curve and the bottom plot shows the contributions of the different noise sources. The darkness of the line in the disturbance contribution plot indicates how strongly a disturbance contributes at a given frequency. It is interesting that the FGS noise is the dominant noise source at low frequency, but that it contributes little to the RMS at high frequencies. The RWA disturbances dominate the region from 5-100 Hz and the cryocooler harmonics are the most important disturbance sources above $100 \mathrm{~Hz}$. We can see that the (cumulative) RMS WFE asymptotes to a value of $933 \mathrm{~nm}$ in Figure 12(a), which exceeds the requirement of 157 $\mathrm{nm}$ as indicated by the dashed horizontal line in the top plot. The error is accumulated in a relatively narrow frequency region from 5-50 Hz. The steps in the cumulative RMS curve can help identify the critical frequencies. These critical frequencies are then represented in the bar chart in Figure 12(b). This chart shows the \% contribution of each critical mode. We can see that the modes at $11.96 \mathrm{~Hz}, 12.72 \mathrm{~Hz}, 26.94 \mathrm{~Hz}$ and $41.18 \mathrm{~Hz}$ together contribute over $50 \%$ to the total RMS WFE. The RWA is the dominant noise source. The second performance metric of interest is the LOS jitter metric as defined in equation (2). It can be interpreted as the average deviation of the image centroid in the $x$ and $y$ direction on the focal plane. Figure 13 summarizes the frequency domain analysis results for the LOS jitter. 

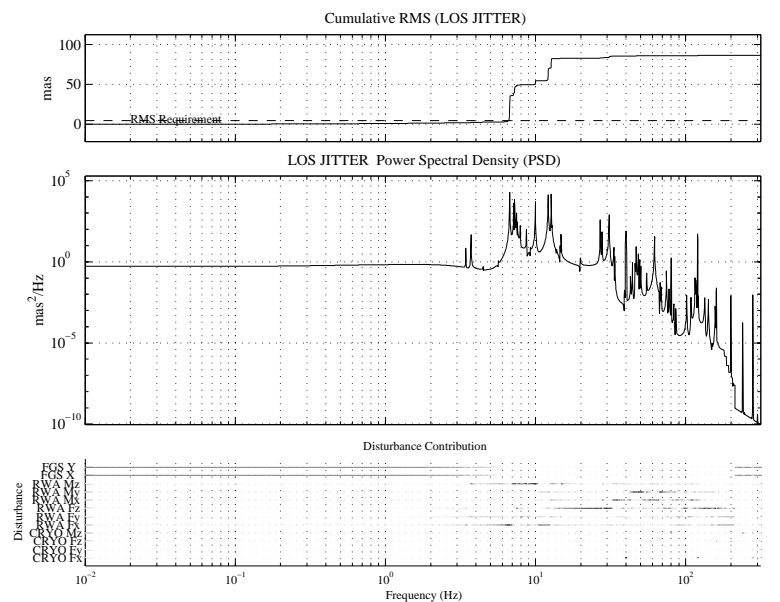

(a)

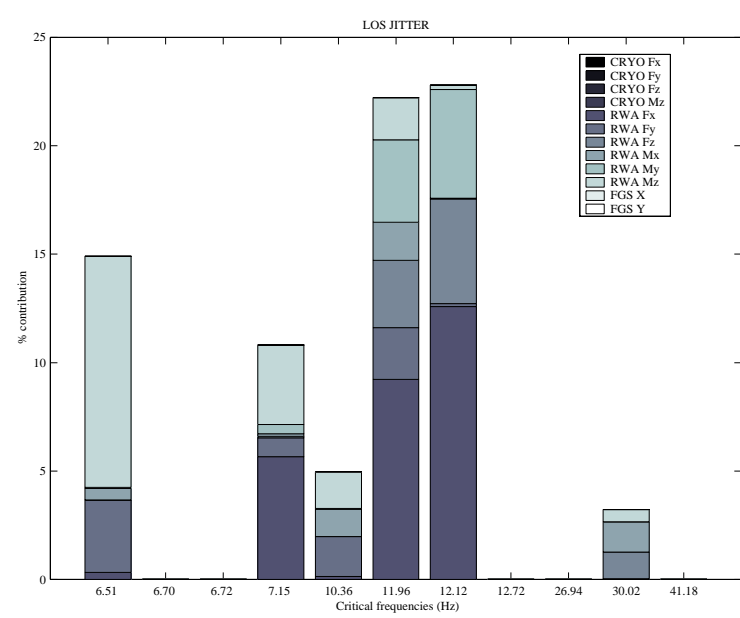

(b)

Figure 13: (a) Baseline PSD analysis results for LOS Jitter; (b) Modal significance chart for LOS Jitter.

The middle subplot of Figure 13(a) shows the PSD of the LOS as a function of frequency in units of $\mathrm{mas}^{2} / \mathrm{Hz}$. The upper plot shows the cumulative RMS curve, which is obtained by integrating under the PSD and taking the square root. The RMS error tends towards a value of RMS LOS $=86.4$ milliarcseconds. The requirement of 4.8 mas is not met based on the baseline assumptions. The critical modes are subsequently extracted and plotted in Figure 13(b). Neither the time domain analysis, nor the Lyapunov analysis is able to provide this level of insight into the system dynamics. From Figure 13(b) we see that just three modes at $6.51,11.96$ and $12.12 \mathrm{~Hz}$ together contribute about $60 \%$ to the total LOS jitter. This is a significant result, since it will allow us to focus on these few modes in order to improve system performance. We must also concentrate our efforts on reducing the contribution of RWA disturbances, since they are the dominant contributor.

The Lyapunov analysis is based on linear systems theory. The state covariance matrix $\Sigma_{q}$ of a linear system, driven by white noise, can be obtained by solving a steady-state Lyapunov equation as follows:

$$
A_{z d} \Sigma_{q}+\Sigma_{q} A_{z d}^{T}+B_{z d} B_{z d}^{T}=0
$$

where $A_{z d}$ and $B_{z d}$ are the system matrices of the white noise $d$ to performance $z$ state space system. The performance covariance matrix $\Sigma_{z}$ is obtained by pre-multiplying $\Sigma_{q}$ with the $C_{z d}$ matrix and by post-multiplying with its transpose:

$$
\Sigma_{z}=C_{z d} \Sigma_{q} C_{z d}^{T}
$$

The variances of the performances $z$ are contained on the main diagonal of $\Sigma_{z}$. Further details of this methodology are shown in in reference [14]. The advantage of the Lyapunov solution is that it provides the exact analytical answer and is immune to frequency vector resolution issues. The disadvantage is that no information about the important modal contributions can be obtained directly. The results from a Lyapunov solution of the baseline case are: RMS WFE $=728 \mathrm{~nm}$ (compared to $933 \mathrm{~nm}$ for PSD analysis) and RMS LOS $=74.4$ mas (compared to 86.4 mas for PSD analysis). We notice that the results do not exactly match the results from the PSD analysis. There are two

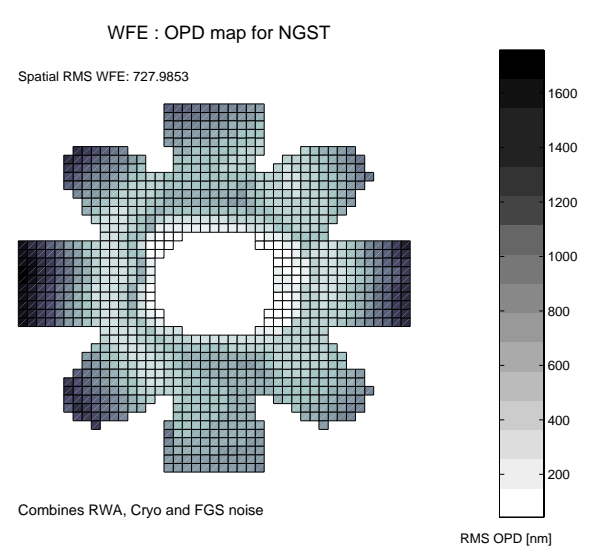

Figure 14: Spatial dynamic RMS WFE distribution across the pupil plane. Distribution appears pixelized due to the rasterization of the spot diagram and 2D-interpolation effects. explanations for this. First, we have had to approximate the disturbance energy with low order filter functions for the RWA, cryocooler and FGS noises for the Lyapunov analysis. Even though our shaping filters might produce the same RMS values as the corresponding disturbance PSD, this does not guarantee that we will obtain the same final result due to the fact that some frequency regions are underpredicted, while others are overpredicted. See Figure 8(c) for an illustration of this effect for the RWA overbounds. Secondly differences arise due to frequency vector resolution issues in the frequency domain. The 
Lyapunov analysis has the advantage that we can cheaply compute the RMS value of the WFE for each individual ray, even if a large number of them (1845) are modeled by MACOS. Because the RMS WFE only represents the spatial RMS value of all rays combined, we can now also look at the spatial distribution of the RMS WFE across the light bundle at the exit pupil. The nominal location of each individual ray is given by the unperturbed spot diagram. The WFE map for NGST is shown in Figure 14. The WFE distribution is not uniform across the aperture. The center segment exhibits the lowest WFE due to its stiff attachment to the hexapod and backup structure. The WFE is larger at the gaps between the center segment and the eight folding segments and generally increases as we go out in a radial direction. The results for the closed-loop NGST model suggest that the performance in terms of RMS WFE and RMS LOS is not met in the baseline case. This conclusion however has to be carefully considered. The most important modeling uncertainties are in the RWA wheel speed distribution and the amount of global damping. Section 5 will analyze how sensitive the performance is to changes in system parameters.

\subsection{Sensor Actuator Topology}

A method has been developed to index the suitability of sets of sensors and actuators for the control of NGST. The indexing is based on each actuator's modal controllability weighted by modal contributions to the performance, and on each sensor's modal observability weighted by modal contributions of the disturbance. Details of the technique are found in [26]. Applying the algorithm to the optical control with the sensor and actuator suite of NGST results in the following index matrix:

$\begin{array}{lllllllll} & \text { T1 } & \text { T2 } & \text { T3 } & \text { RWA1 } & \text { RWA2 } & \text { RWA3 } & \text { FSM1 } & \text { FSM2 } \\ \text { ST1 } & 8.1 e+048 & 8.1 e+049 & 2.2 e+049 & 2.0 e+046 & 3.8 e+041 & 1.1 e+047 & 3.5 e+044 & 8.8 e+042 \\ \text { ST2 } & 6.0 e+047 & 9.5 e+049 & 9.2 e+049 & 4.6 e+041 & 1.0 e+045 & 1.2 e+044 & 1.4 e+044 & 1.9 e+043 \\ \text { ST3 } & 3.3 e+047 & 4.3 e+049 & 7.2 e+049 & 1.1 e+047 & 1.2 e+041 & 3.2 e+048 & 7.5 e+043 & 3.6 e+041 \\ \text { RG1 } & 8.6 e+044 & 9.9 e+045 & 4.2 e+044 & 2.5 e+049 & 7.5 e+045 & 3.7 e+049 & 1.3 e+051 & 2.8 e+049 \\ \text { RG2 } & 2.0 e+048 & 1.5 e+045 & 9.6 e+046 & 7.8 e+045 & 8.2 e+049 & 2.4 e+045 & 5.4 e+050 & 6.1 e+049 \\ \text { RG3 } & 9.1 e+044 & 3.0 e+046 & 1.4 e+045 & 3.7 e+049 & 2.5 e+045 & 1.1 e+051 & 2.9 e+050 & 1.4 e+048 \\ \text { CCD1 } & 2.7 e+058 & 5.4 e+058 & 1.6 e+057 & 3.0 e+058 & 7.8 e+055 & 1.8 e+060 & 1.9 e+086 & 4.5 e+084 \\ \text { CCD2 } & 1.4 e+059 & 2.2 e+060 & 5.5 e+058 & 2.3 e+055 & 7.5 e+058 & 1.8 e+057 & 1.3 e+083 & 8.9 e+085 \\ & & & & & & & \end{array}$

T1-T3 are thrusters, RWA1-RWA3 are reaction wheel assembly axes, FSM1 and FSM2 are the two fast steering mirror axes, ST1- ST3 are three axes of the star trackers, RG1 - RG3 are rate gyroscope sensors, and CCD1 and CCD2 are the two axes of the CCD camera (NIR detector). High values of the index correspond to sensor/actuator combinations which are efficient for control. The channels from FSM1 to CCD1 and from FSM2 to CCD2 are the most efficient for the NGST optical control problem (boxed terms), while the off-diagonal terms (FSM1 to CCD2 and FSM2 to CCD1) are secondary. With information from the sensor / actuator indexing matrix, the baseline controller can be tuned by opening the control channels that have high index values. Control tuning is represented in the diagram of Figure 2 by the 'Optimization' block.

\section{DESIGN TOOLS}

\subsection{Sensitivity Analysis}

The goal of the sensitivity analysis in a broad sense is to understand which parameters of the integrated model most influence the performance metrics, in a narrow sense it seeks to compute the slope of the RMS metrics with respect to modal or physical parameters of the system: $\partial \sigma_{z} / \partial p$. This section creates the link between the critical frequencies of the system found in section 4.1 and a physical understanding of the modes that are involved. Modeshape animations, the modal strain energy distribution and sensitivities for those critical modes are helpful tools. The strain energy is subdivided into the main structural components of NGST and we can understand which groups participate significantly for a given critical mode of the system. For the RMS WFE the most critical modes were at 6.72, 11.96, 12.72, 26.94 and $41.18 \mathrm{~Hz}$. The relative participation of major element groups in the critical modes in terms of strain energy can give valuable information to the designer. The strain energy fraction of the $i$-th group of elements to the total strain energy of the $j$-th mode is defined as $f_{i, j}=\left(\phi_{j, g r o u p}^{T} \mathbf{K}_{\text {group }} \phi_{j, \text { group }}\right) /\left(\phi_{j}^{T} \mathbf{K} \phi_{j}\right)$, where group is the set of degrees-of-freedom that represent the i-th element group for the $\mathrm{j}$-th mode. Depending on the element type (plate, beam, rod) there will be contributions from bending, torsion, shear and tension or compression. The strain energy distribution for the critical modes with respect to the wavefront error (WFE) is shown in Figure 15(a). The figure shows that the secondary tower plays a very important role for the WFE, since it has significant strain energy contributions at virtually all modes. Also, the primary mirror petals contribute a large amount of strain energy with the exception of the mode at $6.72 \mathrm{~Hz}$. This suggests that structural redesign of the secondary tower and the primary mirror petals (backup structure, latches) should be considered. It is also interesting to note that the sunshield flexibility does not significantly impact the WFE. 


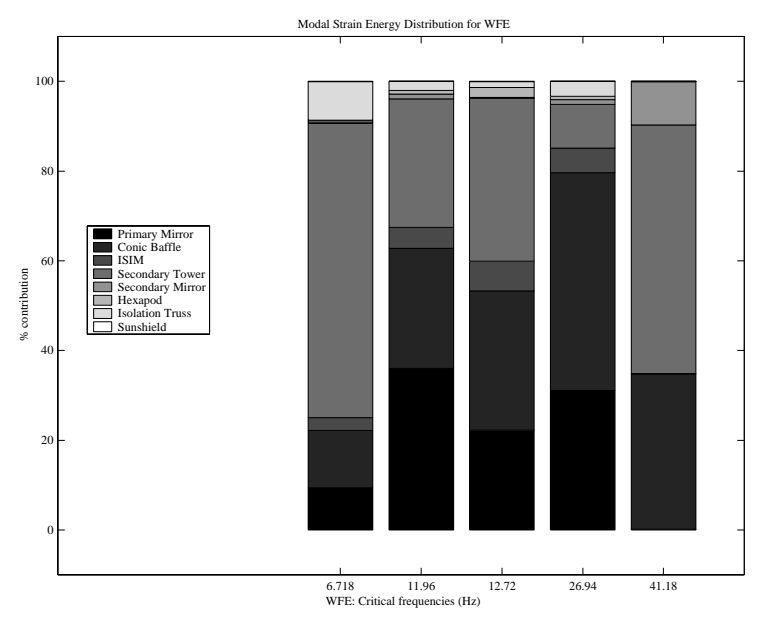

(a)

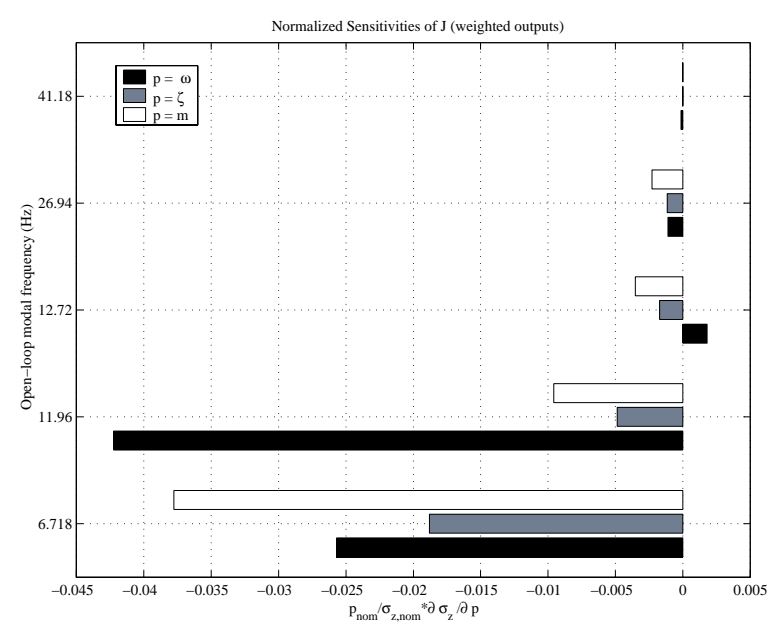

(b)

Figure 15: (a) Strain energy distribution for WFE critical modes; (b) Normalized Modal Sensitivities of RMS WFE (J) for critical modes

The modal parameters of the $i$-th mode are the modal frequency $\omega_{i}$, damping ratio $\zeta_{i}$ and modal mass $m_{i}$. Figure 15(b) shows the results of the modal sensitivity analysis according to [2] for the RMS WFE. We can see that the performance is most sensitive to changes in the modes at 6.718 and $11.96 \mathrm{~Hz}$. As expected, the sensitivity with respect to modal damping $\partial \sigma_{W F E} / \partial \zeta$ is negative, this means that increasing modal damping will decrease the RMS. We should therefore strive to increase the amount of damping in the structure from the current assumed value of $\zeta=0.001$ (see subsection 5.2). Furthermore we see that stiffening these two modes should lead to a decrease in the RMS WFE as well. Since the secondary tower has a significant strain energy contribution to these two modes (see Figure 15(a)) this suggests that stiffening the secondary tower should improve the RMS WFE. In order to confirm this result a physical parameter sensitivity analysis on the secondary tower stiffness parameters should be conducted in the future. We then compute the strain energy distribution for the modes that are critical for the RMS LOS as shown in Figure 16(a). The contribution of the secondary tower is even more important for the pointing (LOS) than for the phasing (WFE). We expect that even slight lateral motion of the secondary mirror will be very visible on the focal plane due to the optical magnification of the system. Again it is not necessarily evident what kind of redesign action should be considered based on strain energy alone. It is not always true that stiffening a structure will lead to better performance.

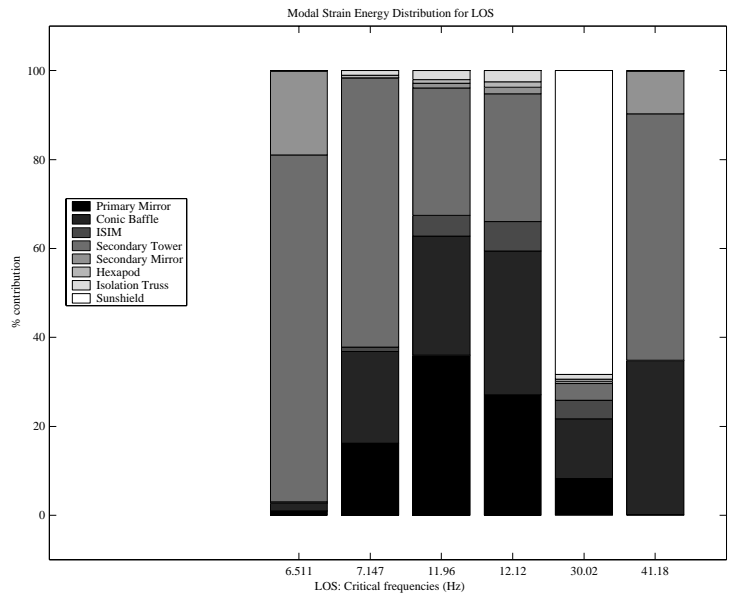

(a)

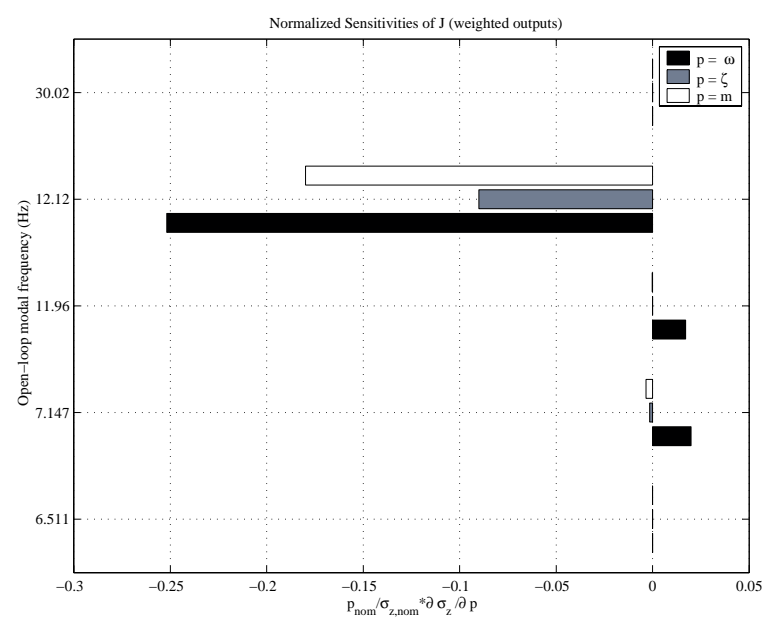

(b)

Figure 16: (a) Strain energy distribution for LOS jitter critical modes; (b) Modal Sensitivities of RMS LOS (J) for critical modes 
Modal sensitivity analysis was performed for the modes that are critical to the LOS (Figure 16(b)). We observe that the mode at $12.12 \mathrm{~Hz}$ has the largest sensitivity w.r.t. to frequency. The sensitivity is normalized, which suggests that a $1 \%$ increase in the modal frequency will result in a $0.25 \%$ decrease of the RMS LOS. This global pitch mode is of significant importance to the pointing of $\mathrm{NGST}^{9}$ and a broad strain energy distribution among the element groups is predicted. This suggests that the $12.12 \mathrm{~Hz}$ mode cannot simply be changed by redesigning a single element since it is a global mode. Disturbance Isolation or more aggressive pointing control seems to be a promising option in this case.

\subsection{Performance Improvement Strategies (Optimization)}

In section 4.1 we established that it was necessary to improve the system performance such that requirements are met and to provide for sufficient design margins. Based on the results of section 5.1 and the controlled structures technology (CST) framework developed by Crawley and coworkers [17] the following system improvements are suggested:

\begin{tabular}{|ll|}
\hline 1. & Use quieter wheels and/or reduce imbalances for existing wheels \\
2. & Implement reaction wheel isolation \\
3. & Structural redesign through stiffening of the secondary tower \\
4. & Addition of passive damping to the system \\
5. & Extend LOS stabilization system bandwidth slightly \\
\hline
\end{tabular}

We analyze suggestions 1 . and 2. in more depth. Using quieter reaction wheels can reduce the disturbance levels significantly. The challenge lies in finding reaction wheels which have sufficient torque and momentum capability without producing large dynamic disturbances. The analysis in Figure 17(a) shows the cumulative RMS levels when four different types of wheels are used. The fourth option is the E-Wheel, where the nominal imbalance level has been reduced by $50 \%$. This is not unrealistic since, according to Bialke, the imbalance levels can be reduced by a factor of 2-3 by carefully balancing and installing the flywheels [11]. This would lead to marginal cost increases, but greatly improve the dynamic performance. Each of the wheel models is based on experimental data and has been modeled using the same input parameters as for the baseline case. The bias wheel speed $R_{o}$ is $1000 \mathrm{RPM}$ and the wheel speed variation is $d R= \pm 1000 \mathrm{RPM}$ with a uniform wheel speed pdf. The results suggest that it is worthwhile to use quieter wheels, but it is insufficient by itself.

Another effective measure is to mechanically isolate the RWA from the rest of the SSM. This isolation can be accomplished passively or actively, depending on the desired corner frequency and rolloff of the transmissibility function. Significant work in this area has been done by Zheng, Haynes and Leonard [27] as well as Spanos, Rahman and Blackwood [28]. In this study the isolator is approximated as a $2^{\text {nd }}$ order system for each axis independently, which neglects cross-axis compliance. Experimental results show that passive isolators work well for high frequencies (above $\sim 100 \mathrm{~Hz}$ ), but that amplification can occur at lower frequencies. Active control needs to be used if we want to achieve corner frequencies below $2 \mathrm{~Hz}$. The corner frequency $f_{i s o}$ was varied in order to determine how isolation can help meet the system performance for NGST.
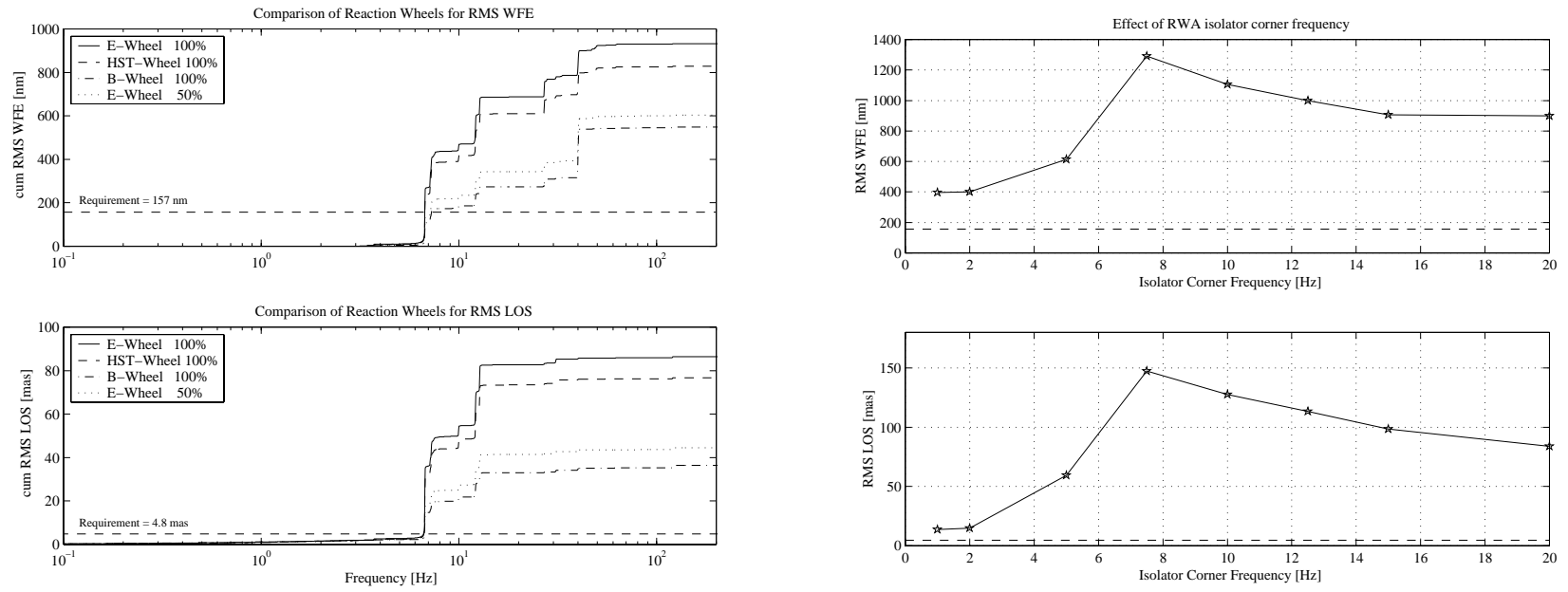

Figure 17: (a) Cumulative RMS curves for four different wheel models. Results are shown as RMS WFE/RMS LOS: HST Wheel (sc=1.0, $828 \mathrm{~nm} / 74.5 \mathrm{mas})$, E-Wheel (sc=1.0, $933 \mathrm{~nm} / 86.4 \mathrm{mas})$, B-Wheel ( $\mathrm{sc}=1.0,549 \mathrm{~nm} / 36.3 \mathrm{mas})$, E-Wheel ( $\mathrm{sc}=0.5,604 \mathrm{~nm} / 44.5 \mathrm{mas})$, $\mathrm{sc}=$ imbalance scale factor ; (b) Trade study for RMS WFE (top) and RMS LOS (bottom) as a function of isolator corner frequency $f_{\text {iso }}$ [Hz].

\footnotetext{
${ }^{9}$ The modeshape of the $12.12 \mathrm{~Hz}$ mode is shown in the lower right hand corner of Figure 4.
} 
Figure 17(b) summarizes the results for the RWA vibration isolation case assuming $20 \%$ isolator damping. We see that an isolator corner frequency between 5 and $15 \mathrm{~Hz}$ does not help but worsens the performance. This can be explained by the fact that the passive (2-parameter) isolator amplifies disturbances near the corner frequency. If this amplification occurs where the disturbance to performance transfer function magnitudes have their maximum values (region between 6 and $12 \mathrm{~Hz}$ ) poor performance results. On the other hand we would expect a more significant improvement between $1 \mathrm{and} 2 \mathrm{~Hz}$, which is not the case. The reason for this phenomenon (details are shown in reference [2] ) is that cryocooler disturbances, specifically the fundamental at $40 \mathrm{~Hz}$, have now become the dominant disturbance sources. The cryocooler disturbance by itself is sufficient to exceed the requirements for the RMS WFE and the RMS $\operatorname{LOS}^{10}$. This leads to the conclusion that further mitigation of cryocooler disturbances along with RWA isolation is necessary. This suggests that a combination of mitigation measures should lead to sufficient performance. A combination case was run, where all of the five proposed performance enhancement steps were incorporated. Table 1 gives a comparison of the disturbance, plant and controls parameters in the baseline case and in the improved design case along with the predicted system performance (computed using frequency-domain approach):

Table 1. Comparison of baseline and improved design parameters and results

\begin{tabular}{|c|c|c|}
\hline Parameter & Baseline Design & Improved Design \\
\hline Bae80K cryocooler attenuation factor & 0.1 & 0.001 \\
\hline E-Wheel Imbalance scale factor for $U_{s}$ and $U_{d}$ & 1.0 & 0.5 \\
\hline RWA Isolator Corner Frequency $f_{\text {iso }}$ & None & $2 \mathrm{~Hz}$ \\
\hline FGS Integration time & $33.3 \mathrm{msec}$ & $20 \mathrm{msec}$ \\
\hline Modal Damping Ratio & 0.001 & 0.01 \\
\hline SM Tower Blade Area moment of inertia $I_{l}$ & $2.7778 \mathrm{e}-7$ & $5.0 \mathrm{e}-6$ \\
\hline Performance $z_{1}:$ RMS WFE (req: $157 \mathrm{~nm}$ ) & $933 \mathrm{~nm}$ & $21.2 \mathrm{~nm}$ \\
\hline Performance $z_{2}:$ RMS LOS (req: 4.8 mas) & 86.4 mas & 3.20 mas \\
\hline
\end{tabular}

The improved design meets requirements with a predicted RMS WFE of $21.22 \mathrm{~nm}$ (42.6 dB margin) and RMS LOS of 3.204 milli-arcseconds $(0.2 \mathrm{~dB}$ margin). This suggests that it will likely be more difficult to meet the LOS jitter than the WFE requirement for NGST. The largest contributor to the RMS LOS has become the FGS noise in the improved design, since we have reduced the mechanical noise sources below the FGS noise level. This is desirable, since the pointing performance is now limited by the brightness of an "opportunistic" guide star and not by the imperfections of the hardware. An interesting trade becomes apparent, when we shorten the FGS integration time. As $\mathrm{T}_{\mathrm{INT}}$ is decraesed the system requires brighter guide stars, which in turn leads to a larger field-of view (FOV). This can be accomodated with a larger focal plane or a change in the telescope f/\#. In Table 1 we have found a combination of performance enhancements that will bring the system into compliance with the performance requirements stated in section 1. It is true, however, that the combination presented in this subsection is not unique. This motivates the need for an isoperformance analysis tool as discussed in the next subsection.

\subsection{Isoperformance Analysis}

Once a point design (Table 1, right column) has been found that meets the dynamic requirements, we are interested in finding combinations of important system parameters that will results in the equivalent RMS performance. For a precision telescope such as NGST for example the pointing requirement could be met with a HST-like rigid spacecraft body and OTA, whereby the ACS performs body-pointing with high-precision rate gyros and a dedicated FGS. On the other hand the same pointing requirement can be met with a low-precision ACS but a fine pointing loop as suggested for the Yardstick design. Isoperformance is a promising methodology, which uses the nullspace of the sensitivity matrix, whose entries are formed by the sensitivities $\partial \sigma_{z, i} / \partial p_{j}$, where $\sigma_{z, i}$ is the $\mathrm{i}$-th performance RMS value and $p_{j}$ is the $\mathrm{j}$-th parameter of the system. Exploring this nullspace is equivalent to following the contours of equal pressure (isobars) on a weather map. This allows to treat the performance $z$ as a constraint, while trading important

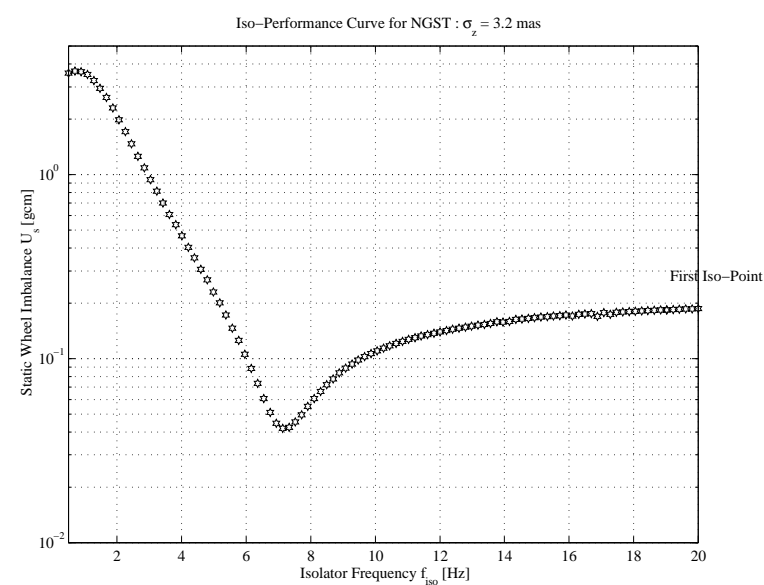

Figure 18: NGST Preliminary isoperformance analysis for static wheel imbalance Us [ $\mathrm{gcm}]$ versus isolator corner frequency $[\mathrm{Hz}]$ at the RMS LOS $=3.2$ mas level.

\footnotetext{
${ }^{10}$ It is unlikely that a noisy Sterling cooler will be chosen for NGST. Current development efforts point toward a turbo Brayton cooler.
} 
system parameters with respect to each other. Figure 18 shows the results of a preliminary isoperformance analysis for NGST (assumption: have only one reaction wheel running at $430 \mathrm{RPM}$ as disturbance input). The static imbalance $U_{s}$ of a reaction wheel is traded with the isolator corner frequency $f_{\text {iso, }}$, while holding the RMS LOS constant at the 3.2 mas level found in the previous subsection. The results from Figure 16(b) are corroborated, since an isolator corner frequency in the 5-15 Hz range requires a tighter requirement on the static imbalance $U_{s}$. Even though the model assumptions in this preliminary analysis are different from the baseline model in Figure 3, we see that at an isolator frequency of $2 \mathrm{~Hz}$ a static imbalance of $U_{s}=0.9 \mathrm{gcm}$ will achieve the 3.2 mas performance. This is a reasonable prediction, given the fact that the experimental static imbalance of the E-wheel from subsection 2.4 was found to be $0.716 \mathrm{gcm}$. Isoperformance analysis is a promising emerging tool for subsystem requirements definition and dynamics and controls error budgeting.

\section{CONCLUSIONS AND RECOMMENDATIONS}

The two key metrics in this paper are the root-mean-square wavefront error (RMS WFE) and the line-of-sight jitter rootmean-square error (RMS LOS). It is important to assess the impact of the expected micro-vibration environment on the scientific performance before design, construction and launch. The DOCS framework presented here provides a set of useful analysis and design tools for the conceptual and preliminary design phase of a precision opto-mechanical space system such as NGST. An integrated model including a reaction wheel assembly (RWA), cryocooler vibrations and guide star sensor noise was created. The predicted baseline performance (using the PSD-based approach) was a RMS WFE value of 933 nm and a RMS LOS value of 86.4 mas, which did not meet the requirements of $\sigma_{\mathrm{WFE}}=157 \mathrm{~nm}$ and $\sigma_{\mathrm{LOS}}=4.8 \mathrm{mas}$. It was also found that the RWA is the dominant noise contributor and that the critical modes for the system lie in the region between 5 and $50 \mathrm{~Hz}$. The performance improvements to the baseline design include increasing the LOS stabilization bandwidth, stiffening the secondary mirror tower, using a passive 2-Hz RWA isolator, adding passive damping, using a quieter cryocooler and finally reducing the imbalances of the reaction wheels in the first place. It has been shown quantitatively that the combination of these incremental changes brings the system into compliance with the requirements. The resulting performance for the improved design was predicted to be RMS WFE $=21.2 \mathrm{~nm}$ and RMS LOS $=3.204$ mas, respectively. Thus a true system level improvement has been carried out, without attempting to optimize each subsystem individually.

The following activities are recommended for future research:

- Apply the uncertainty analysis framework to the NGST analysis. It was shown in [2] that model assumptions such as modal damping can have a large effect on the RMS results. An uncertainty analysis would determine which parameters are uncertain and within what bounds. This would allow to put "error bars" on the results of the performance assessment.

- Perform a physical parameter sensitivity analysis for NGST. The physical parameters of interest are part of the disturbance models (e.g. wheel speed (RPM) ranges, imbalance levels), structural plant (e.g. blade stiffness SM tower), optics (e.g. focal length of system, conical section properties of mirrors) and the controller (e.g. FSM controller bandwidth).

- Compute second derivatives (curvatures) of the RMS. This would allow predicting the range of validity of the sensitivities (slope). It is expected that some parameters behave very well, but other parameters can flip signs and show non-linear behavior depending on the specific model assumptions.

- Exercise a gradient-based optimization tool shown in Figure 2 in order to optimize the performance RMS, while each parameter is given an allowable bound. A cost function could include RMS, control effort, system mass, system cost and stability margins.

- Multivariable isoperformance analysis based on physical parameter sensitivities. This would allow finding alternate designs (based on the same topology as the nominal design) in the trade space of "free" design parameters that will lead to the same performance in terms of RMS WFE and RMS LOS.

\section{ACKNOWLEDGMENTS}

This research has been supported by NASA's Goddard Space Flight Center under RTOP grant No. NAG5-6079 and under research grant NAG5-7839. The following individuals at GSFC have contributed to this work through their technical suggestions and support: Gary Mosier, Bill Hayden, Mike Femiano, Kong Ha, Richard Burg and Ed James. The following individuals at the Jet Propulsion Laboratory (JPL) were helpful in answering specific questions relating to IMOS, structural modeling, optical modeling and MACOS: Andy Kissil and Dave Redding. The support and previous work of Dr. Homero Gutierrez and Mrs. Becky Masterson is also gratefully acknowledged. 


\section{REFERENCES}

[1] de Weck, O., Miller D.W., Gutierrez H., "Structural Dynamics and Controls for NGST - A preliminary study", presented at the $34^{\text {th }}$ Liège International Astrophysics Colloquium in Liège, Belgium, 15-18 June 1998

[2] de Weck, O., "Integrated Modeling and Dynamics Simulation for the Next Generation Space Telescope", S.M. Thesis, Massachusetts Institute of Technology, Department of Aeronautics and Astronautics, June 1999

[3] NASA GSFC Study team, "The Next Generation Space Telescope"- A presentation to the NGST study office, NASA Goddard Space Flight Center, August 21, 1996

[4] Bely P., Burg, R. Perrygo C., "NGST Monograph No. 1" NGST Yardstick Mission, NASA Goddard Space Flight Center , Draft version, 1997

[5] H.S. Stockman (editor) et al., "Next Generation Space Telescope - Visiting a Time when Galaxies were Young", The NGST Study Team, The Association of Universities for Research in Astronomy, Inc., June 1997

[6] Redding D. e a. "Wavefront Sensing and Control for the Next Generation Space Telescope", SPIE Conference on Astronomical Telescopes and Instrumentation, Kona, 1998

[7] Mosier, Gary, Mike Femiano and Kong Ha, Goddard Space Flight Center, Redding, Dave, JPL, National Aeronautics and Space Administration, “An Integrated Modeling Environment for Systems-level Performance Analysis of the Next Generation Space Telescope", SPIE Conference on Astronomical Telescopes and Instrumentation, Kona, 1998

[8] Mosier G., Ha K., Femiano M., "NGST Slew Algorithm Description”, GSFC Internal Memorandum, May 28, 1998

[9] Kissil A., " Description of NGST Finite Element Models”, Internal Memorandum, 1998

[10] Redding, D.C., Breckenridge W.G., "Optical modeling for Dynamics and Control Analysis", AIAA-90-3383, 1990

[11] Bialke, Bill, "A compilation of reaction wheel induced spacecraft disturbances", $20^{\text {th }}$ Annual Annual American Astronautical Society Guidance and Control Conference, paper 97-038 Breckenridge, Colorado, February 5-9, 1997

[12] Castles S. e.a., "NASA/GSFC Cryocooler Development Program”, GSFC Internal Memorandum, 1996

[13] Collins, S. A., "Multi-Axis Analog Adaptive Feedforward Cancellation of Cryocooler Vibration”, Ph.D. thesis, Massachusetts Institute of Technology, Department of Aeronautics and Astronautics, 1994

[14] Gutierrez, H.,"Performance Assessment and Improvement of Precision Structures during Conceptual Design", Ph.D. Thesis, Massachusetts Institute of Technology, Department of Aeronautics and Astronautics, February 1999

[15] JPL Publication 98-12 “Integrated Modeling of Optical Systems User's manual”, Release 4.0, National Aeronautics and Space Administration, Jet Propulsion Laboratory, California Institute of Technology, Pasadena, California, September 19, 1998

[16] Mosier, G., "Analysis of Solar Pressure and C.M.-C.P. Offset on Momentum Unloading Distribution, NGST Systems Analysis Memorandum , NASA Goddard Space Flight Center, July 17, 1997

[17] Crawley, E., Hall S., "Dynamics of Controlled Structures", 16.243 Course Notes, Space Engineering Research Center (SERC), Massachusetts Institute of Technology, 1991

[18] Mosier,G.E., Femiano M.,NASA/GSFC, Kong Ha, Jackson \& Tull, Burg R., JHU, "Dynamics and Controls”, Presentation at Modeling Peer Review, JPL, January 22, 1998

[19] Sparr L.,Boyle R., Cory R., Connors F., James E., Fink R., Arillo V., Marketon J., "NASA/GSFC Cryocooler Test Program Results", Memorandum, NASA Goddard Space Flight Center, 1993

[20] Melody, J.W., "Discrete Frequency and Broadband Reaction Wheel Disturbance Models", JPL Interoffice Memorandum, 3411-95200csi, June 1, 1995

[21] Masterson R., "Development and Validation of Empirical and Analytical Reaction Wheel Disturbance Models", S.M. thesis, Massachusetts Institute of Technology, June 1999

[22] Gutierrez, H., "Stochastic Frequency-domain Disturbance Model of Multiple Reaction Wheels of Arbitrary Orientation", Memorandum to Jet Propulsion Laboratory, February 13, 1998

[23] Gutierrez, H., Miller, D.W., "Disturbance Analysis of Integrated Structural/Optical Models with Uncertainties", Proceedings of the American Control Conference, Philadelphia, PA, June, 1998

[24] Breault Research Organization, "Modeling and Analysis for Controlled Optical Systems", MACOS User Manual, Version 2.4, April 1, 1997

[25] Curtis A. (editor), Miller, D.W. e.a., “Terrestrial Planet Finder Architecture Design Report”, 16.89 Space Systems Engineering, Massachusetts Institute of Technology, May 1999

[26] Mallory, Gregory. J. W., "Development and Experimental Validation of Direct Controller Tuning for Spaceborne Telescopes", Ph.D. dissertation, Massachusetts Institute of Technology, Department of Aeronautics and Astronautics, 2000.

[27] Geng, Zheng; Haynes, Leonard S ,'Multiple degree-of-freedom active vibration control using a Stewart platform”,International Conference on Adaptive Structures, 3rd, San Diego, CA, Nov. 9-11, 1992, Proceedings (A94-20579 04-63), Lancaster, PA, Technomic Publishing Co., Inc., 1993, p. 499-513

[28] Spanos J, Rahman Z., Blackwood G., “A Soft 6-axis Acive Vibration Isolator”, American Control Conference, Seattle, WA, June 21-23, 1995

[29] Mosier G., Femiano M., Ha K., Bely P., Burg R., Redding D., Kissil A., Rakoczy J and Craig L, "Space Telescope Science Fine Pointing Control for a Next Generation Space Telescope", Proceedings of SPIE Astronomical Telescopes and Instrumentation Conference, Kona, Hawaii, 1998 